DESY $10-156$

SFB/CPP-11-51

\title{
One-loop lattice artifacts of a dynamical charm quark
}

\author{
$\bar{A} L P H A$ \\ Andreas Athenodorou and Rainer Sommer \\ NIC, DESY, Platanenallee 6, 15738 Zeuthen, Germany
}

\begin{abstract}
For a few observables in $\mathrm{O}(a)$ improved lattice $\mathrm{QCD}$, we compute discretization effects arising from the vacuum polarization of a heavy quark at one-loop order. In particular, the force between static quarks, the running coupling in the Schrödinger functional and a related quantity, $\bar{v}$, are considered. Results show that the cutoff effects of a dynamical charm quark are typically smaller than those present in the pure gauge theory. This perturbative result is a good indication that dynamical charm quarks are feasible already now.
\end{abstract}

Key words: Lattice QCD, Charm quarks, cutoff effects, Lattice perturbation theory PACS: 12.38.Gc, 14.65.Dw, 12.38.Bx, 11.10.Gh

September 2011 


\section{Introduction}

Some collaborations are starting to include charm quark vacuum polarization in simulations of lattice QCD [1 4]. Clearly the motivation is to exclude noticeable corrections due to charm quark loops, especially when processes are considered where intermediate to large momentum transfers are relevant. A particular such case is the extraction of the fundamental parameters of QCD, the $\Lambda$-parameter and quark masses. A nonperturbative computation of these parameters needs a control of the theory over a large energy range, in the ideal case by a step scaling method [5]. If such a computation is only based on simulations of the three-flavor theory, the connection to the four and more flavor theory has to be done perturbatively, without non-perturbative control. The ALPHA-collaboration has therefore started a computation in the four flavor theory [3]. So far, only the running coupling in the massless Schrödinger functional scheme has been considered. In order to set the scale, i.e. determine the energy scale in GeV, physical observables in the massive theory have to be computed in addition and matched to experiment. The question then arises whether this is feasible with the lattice spacings of around $0.1 \mathrm{fm} \lesssim a \lesssim 0.05 \mathrm{fm}$ which are accessible today.

The main reason to worry is that the charm quark mass in lattice units, $m_{\mathrm{c}} a$, is as large as $1 / 2$ in this situation 1 In the valence quark sector it has been found that up to such values cutoff effects in the $\mathrm{O}(a)$-improved theory can be sizeable. They do approximately show the expected form, quadratic in $a$ [10 12], but it is also known that above $a m=1 / 2$ the Symanzik analysis of cutoff effects breaks down and Symanzik $\mathrm{O}(a)$-improvement ceases to be useful [13, 14]. This knowledge has mainly been accumulated in theories with a quenched charm quark; the large effects are due to the propagation of a valence charm quark. A discussion of the cutoff effects of vacuum polarization contributions of heavy quarks has been given in Ref. [15], again emphasizing the breakdown of the Symanzik expansion 2

In this paper we take a further step towards answering the question of the size of cutoff effects in charm quark vacuum polarization effects. We consider the leading such effects in perturbation theory, namely we expand a few observables in the renormalized coupling and examine the dependence of the first non-trivial expansion coefficient on the lattice spacing and the mass of the quark. We make use of our previous work with H. Panagopoulos [17, extracting the cutoff effects in the force between static quarks and illustrate further the cutoff effects present in the fermionic contribution to the Schrödinger functional coupling [15]. Obviously the size of these effects is nonuniversal, depending on all details of the regularization. Precise statements refer to the $\mathrm{O}(a)$ improved theory that we use. However, this does provide an example how much cutoff effects grow or do not with the mass of a dynamical quark. A shortcoming of our

\footnotetext{
${ }^{1}$ The value of $m_{\mathrm{c}}$ does of course depend on the renormalization scheme and the renormalization scale, but for qualitative questions which we discuss here, this is not important.

${ }^{2}$ Note in this context that the large effects found in 2 are not of relevance in a theory with exact flavor symmetry. They can't provide a guidance for the $\mathrm{O}(a)$-improved theory as defined in [16].
} 
investigation is that we just use perturbation theory. However concerning the qualitative picture we do not think this restriction is too severe: the dominant contributions of charm quark loops is to processes with typical momenta of the size of the charm mass. While such momenta are not large enough to expect high precision from perturbation theory, we expect the qualitative features to be quite reliable.

\section{Lattice formulation, renormalization and cutoff effects}

We start by recalling the most important features of the $\mathrm{O}(a)$-improved theory which are relevant in the following. Its definition is discussed in detail in Ref. [16], whose notation we use. Note that in principle the structure of the improved theory is more complicated when all quark masses are considered [18], but this is irrelevant here, where we consider only one-loop effects.

In Wilson's regularization, the total action $S=S_{g}+S_{f}$ is given by

$$
\begin{aligned}
S_{g}[U] & =\frac{1}{g_{0}^{2}} \sum_{p} w(p) \operatorname{tr}\{1-U(p)\}, \\
S_{f}[U, \bar{\psi}, \psi] & =a^{4} \sum_{x} \sum_{i=1}^{N_{\mathrm{f}}} \bar{\psi}_{i}\left(D+m_{0, i}\right) \psi_{i} .
\end{aligned}
$$

The gauge field action $S_{g}$ is a sum over all oriented plaquettes $p$ on the lattice, with the parallel transporters $U(p)$ around $p$. The weights $w(p)$ are set to unity for now and we consider an infinite lattice. The Dirac operator is

$$
D=\frac{1}{2} \sum_{\mu=0}^{3}\left\{\gamma_{\mu}\left(\nabla_{\mu}^{*}+\nabla_{\mu}\right)-a \nabla_{\mu}^{*} \nabla_{\mu}\right\}+c_{\mathrm{sw}} \frac{i a}{4} \sum_{\mu, \nu=0}^{3} \sigma_{\mu \nu} \hat{F}_{\mu \nu}
$$

with $\nabla_{\mu}$ and $\nabla_{\mu}^{*}$ the forward and backward covariant derivatives respectively. The improvement term involving the lattice approximation of the field strength $\hat{F}_{\mu \nu}$ (see [16] for its precise definition) has a coefficient [19]

$$
c_{\mathrm{sw}}=1+\mathrm{O}\left(g_{0}^{2}\right) .
$$

The discussion of cutoff effects only makes sense after a renormalization of the theory and the cutoff effects do depend on the renormalization conditions. Here we are interested in perturbation theory and we first choose massless renormalization schemes with a renormalization scale $\mu$.

\subsection{Massless renormalization schemes}

At the required order, the renormalized coupling and quark masses are given by

$$
\bar{g}^{2}(\mu)=\tilde{g}_{0}^{2} Z_{\mathrm{g}}\left(\tilde{g}_{0}^{2}, a \mu\right), \quad\left(m_{i}\right)_{\mathrm{R}}(\mu)=\widetilde{m}_{\mathrm{q}, i}+\mathrm{O}\left(g_{0}^{2}\right),
$$


in terms of the improved bare coupling and improved quark mass

$$
\tilde{g}_{0}^{2}=g_{0}^{2}\left(1+b_{\mathrm{g}}^{(1)} g_{0}^{2} a \sum_{i=1}^{N_{f}} m_{0, i}\right)+\mathrm{O}\left(g_{0}^{6}\right), \quad \widetilde{m}_{\mathrm{q}, i}=m_{0, i}\left(1-\frac{1}{2} a m_{0, i}\right),
$$

where [15, 16]

$$
b_{\mathrm{g}}^{(1)}=0.01200(2) \text {. }
$$

We remark once more that at higher orders in perturbation theory, the structure of renormalization and improvement is more complicated in the case of non-degenerate quark masses [18].

Often one considers observables whose perturbative expansion in $\bar{g} \overline{\mathrm{MS}}$ is known in the continuum theory. It is then natural to renormalize in the $\overline{\mathrm{MS}}$ scheme. For clarity this is done in two steps. First we introduce the coupling in the lattice minimal subtraction scheme, defined by subtracting order by order in perturbation theory just a polynomial in $\log (a \mu)$ without a constant part. For our purposes this means

$$
\bar{g}_{\text {lat }}^{2}(\mu)=\tilde{g}_{0}^{2} Z_{\text {lat }}\left(\tilde{g}_{0}^{2}, a \mu\right), \quad Z_{\text {lat }}\left(\tilde{g}_{0}^{2}, a \mu\right)=1-2 b_{0} \tilde{g}_{0}^{2} \log (a \mu)+\mathrm{O}\left(\tilde{g}_{0}^{4}\right),
$$

with $b_{0}=\frac{1}{(4 \pi)^{2}}\left(\frac{11}{3} N_{c}-\frac{2}{3} N_{f}\right)$ and $N_{c}$ the number of colors.

Let us now consider an observable 3 which depends on a single length scale $r$ in addition to the masses $\left(m_{i}\right)_{\mathrm{R}}$ and which has an expansion 4

$$
O=g_{0}^{2}+O^{(1)}(\mathbf{z}, a / r) g_{0}^{4}+\ldots,
$$

with $\mathbf{z}=\left(z_{1}, \ldots, z_{N_{\mathrm{f}}}\right)$ and $z_{i}=\left(m_{i}\right)_{\mathrm{R}} \cdot r$. The observables that we use below are exactly of this form. After inserting $g_{0}^{2}=\bar{g}_{\text {lat }}^{2}+\left[2 b_{0} \log (a \mu)-b_{\mathrm{g}}^{(1)} a \sum_{i=1}^{N_{f}} m_{0, i}\right] \bar{g}_{\text {lat }}^{4}+\ldots$ the renormalized expression has a unique split

$$
O=\tilde{O}_{\text {cont }}\left(r \mu, \mathbf{z}, \bar{g}_{\text {lat }}^{2}(\mu)\right)\left(1+\tilde{\delta}_{O}\left(r \mu, \mathbf{z}, \bar{g}_{\text {lat }}^{2}(\mu), a / r\right)\right),
$$

into a continuum piece and a lattice artifact

$$
\tilde{\delta}_{O}\left(r \mu, \mathbf{z}, \bar{g}_{\text {lat }}^{2}(\mu), a / r\right)=\tilde{\delta}_{O}^{(0)}(r \mu, \mathbf{z}, a / r)+\tilde{\delta}_{O}^{(1)}(r \mu, \mathbf{z}, a / r) \bar{g}_{\text {lat }}^{2}(\mu)+\ldots,
$$

with $\tilde{\delta}_{O}\left(r \mu, \mathbf{z}, \bar{g}_{\text {lat }}^{2}(\mu), 0\right)=0$. We may now switch renormalization to the $\overline{\mathrm{MS}}$ scheme via the finite scheme transformation (independent of the lattice spacing)

$$
\begin{aligned}
\bar{g}_{\text {lat }}^{2}(\mu) & =\bar{g}_{\overline{\mathrm{MS}}}^{2}(\mu)-\frac{c_{1}^{\text {lat }, \overline{\mathrm{MS}}}}{4 \pi} \bar{g} \frac{4}{\mathrm{MS}}(\mu)+\mathrm{O}\left(\bar{g} \frac{6}{\mathrm{MS}}(\mu)\right), \\
c_{1}^{\text {lat }, \overline{\mathrm{MS}}} & =c_{1, g}+N_{\mathrm{f}} c_{1, f}, \\
c_{1, g} & =-\frac{\pi}{2 N_{\mathrm{c}}}+2.135730074078457(2) N_{\mathrm{c}}, \quad c_{1, f}=-0.39574962(2),
\end{aligned}
$$

\footnotetext{
${ }^{3}$ By observable we simply mean a quantity which is free of divergences after coupling renormalization and quark mass renormalization.

${ }^{4}$ In general one has $\bar{O}=\bar{O}^{(0)}(\mathbf{z}, a / r)+\bar{O}^{(1)}(\mathbf{z}, a / r) g_{0}^{2}+\ldots$ for a single scale observable with a regular expansion in $g_{0}^{2}$ made dimensionless using an appropriate power of $r$. Assuming $\bar{O}^{(1)}$ to be non-zero, one may then form $O=\left(\bar{O}-\bar{O}^{(0)}\right) / \bar{O}^{(1)}$, which has the interpretation of a renormalized coupling and the expansion eq. (2.9).
} 
where the coefficients are known from Refs. [20 22] and Refs. [15, 23]. We then end up with the expansion of the relative lattice artifacts

$$
\begin{aligned}
\delta_{O}\left(r \mu, \mathbf{z}, \bar{g}_{\overline{\mathrm{MS}}}^{2}(\mu), a / r\right) & \left.\equiv \frac{O-O_{\mathrm{cont}}}{O_{\mathrm{cont}}}\right|_{\bar{g}_{\overline{\mathrm{MS}}},\left(m_{i}\right)_{\mathrm{R}}} \\
& =\delta_{O}^{(1)}(r \mu, \mathbf{z}, a / r) \bar{g}_{\overline{\mathrm{MS}}}^{2}(\mu)+\ldots
\end{aligned}
$$

Our notation for the left hand side specifies explicitly how the theory is renormalized: $\bar{g} \overline{\overline{\mathrm{MS}}}(\mu)$ and $\left(m_{i}\right)_{\mathrm{R}}$ are held fixed as the continuum limit is approached.

A possibly confusing point is that these lattice artifacts are intrinsically perturbative, because the way the theory is renormalized (by minimal subtraction) has no non-perturbative extension. The artifacts $\delta_{O}^{(i)}$ therefore can not be regarded as perturbative expansion coefficients of artifacts which appear in a non-perturbative solution of the theory by a Monte Carlo computation. However, combinations of the above defined $\delta^{(i)}$ from different observables yield the expansion coefficients of true non-perturbative artifacts. Let us briefly illustrate this.

As an example we choose to renormalize the theory through the Schrödinger functional coupling $\bar{g}_{\mathrm{SF}}(L)$ [24] for massless quarks [15]. Non-perturbatively this means that for each value of $a$ the bare coupling is chosen such that $\bar{g}_{\mathrm{SF}}(L)$ is held fixed. We follow the literature and keep as argument of $\bar{g}_{\mathrm{SF}}$ the length scale $L$, related to the energy renormalization scale as $\mu=1 / L$. Since the Schrödinger functional coupling is an observable, its perturbation theory in terms of $\bar{g}_{\overline{\mathrm{MS}}}^{2}$ is just as described above,

$$
\bar{g}_{\mathrm{SF}}^{2}(L)=\left[\bar{g}_{\overline{\mathrm{MS}}}^{2}(\mu)+c_{1}^{\mathrm{SF}, \overline{\mathrm{MS}}} \bar{g}_{\overline{\mathrm{MS}}}^{4}(\mu)+\ldots\right] \cdot\left[1+\delta_{\mathrm{SF}}^{(1)}(a / L) \bar{g}_{\overline{\mathrm{MS}}}^{2}(\mu)+\ldots\right] .
$$

Combining this expansion with eq. (2.16) we have

$$
\begin{aligned}
\delta_{O_{\mathrm{SF}}}\left(r \mu, \mathbf{z}, \bar{g}_{\mathrm{SF}}^{2}(L), a / r\right) & \left.\equiv \frac{O-O_{\mathrm{cont}}}{O_{\mathrm{cont}}}\right|_{\bar{g}_{\mathrm{SF}},\left(m_{i}\right)_{\mathrm{R}}} \\
& =\left[\delta_{O}^{(1)}(r \mu, \mathbf{z}, a / r)-\delta_{\mathrm{SF}}^{(1)}(a / L)\right] \bar{g}_{\mathrm{SF}}^{2}(L)+\ldots
\end{aligned}
$$

The combination $\delta_{O}^{(1)}(r \mu, \mathbf{z}, a / r)-\delta_{\mathrm{SF}}^{(1)}(a / L)$ does describe the asymptotic behavior of the non-perturbatively defined left hand side at small coupling, i.e. small $r$ and $L$.

In this paper we are interested in the cutoff effects induced by heavy quark vacuum polarization. We take observables without valence quarks ("pure gauge observables"), split all cutoff effects into a gluonic one and those due to the different quarks,

$$
\delta_{O}^{(1)}(r \mu, \mathbf{z}, a / r)=\delta_{O}^{(1, g)}(r \mu, a / r)+\sum_{i=1}^{N_{f}} \delta_{O}^{(1, f)}\left(r \mu, z_{i}, a / r\right)
$$

and are interested in particular, whether $\delta_{O}^{(1, f)}(r \mu, z, a / r)$ is large when $z$ is large, as it may be the case for a charm quark. Holding the mass-less Schrödinger functional 
coupling fixed in taking the continuum limit, the relevant contribution to the cutoff effect is then

$$
\delta_{O}^{(1, f)}(r \mu, z, a / r)-\delta_{\mathrm{SF}}^{(1, f)}(a / L) \approx \delta_{O}^{(1, f)}(r \mu, z, a / r) .
$$

Here we have used the fact that for the standard Schrödinger functional coupling and massless quarks the contribution $\delta_{\mathrm{SF}}^{(1, f)}(a / L)$ is very small [15. In Sect. 3 we will, therefore, simply discuss $\delta_{O}^{(1, f)}(r \mu, z, a / r)$ choosing as an observable the force between static quarks.

\subsection{Massive renormalization schemes}

While a massless renormalization scheme is attractive since it keeps the renormalization group equations simple, it is not the most convenient choice for non-perturbative computations in QCD with a charm quark. The charm quark's mass is larger than the typical QCD scale. It therefore has reduced vacuum polarization effects which is most efficiently implemented by renormalizing at the finite mass of the charm quark. (Of course the computation of true mass-effects is most easily done in a massless scheme.)

A physical massive scheme is defined by picking a specific observable $O=O_{0}$ with the expansion eq. (2.9) and defining the coupling in the massive scheme

$$
\bar{g}_{m}^{2}\left(\mu, \mathbf{m}_{\mathrm{R}}\right) \equiv O_{0}=g_{0}^{2}+O_{0}^{(1)}(\mathbf{z}, a / r) g_{0}^{4}+\ldots,
$$

with $\mu=1 / r_{0}$. In principle this has to be supplemented by a condition for $\mathbf{m}_{\mathrm{R}}$, but we do not need that at the perturbative order considered here.

Straight forwardly, as in eq. (2.18), we then have for a different observable $O$ or the same one at a different length scale $r$,

$$
\begin{aligned}
\delta_{O_{\mathrm{m}}}\left(r / r_{0}, \mathbf{z}, \bar{g}_{\mathrm{m}}^{2}\left(\mu, \mathbf{m}_{\mathrm{R}}\right), a / r\right) & \left.\equiv \frac{O-O_{\text {cont }}}{O_{\text {cont }}}\right|_{\bar{g}_{\mathrm{m}},\left(m_{i}\right)_{\mathrm{R}}} \\
& =\left[\delta_{O}^{(1)}\left(r \mu, r \mathbf{m}_{\mathrm{R}}, a / r\right)-\delta_{O_{0}}^{(1)}\left(r \mu, r_{0} \mathbf{m}_{\mathrm{R}}, a / r_{0}\right)\right] \bar{g}_{\mathrm{m}}^{2}\left(\mu, \mathbf{m}_{\mathrm{R}}\right)+\ldots
\end{aligned}
$$

The cutoff effects in the massive scheme are just given as a combination of the ones in the massless scheme.

\section{The force between static quarks}

As a first observable we consider the force $F(r)$ between static quarks at a distance $r$. In the continuum it is defined in terms of the static potential as $F(r)=\frac{d}{d r} V(r)$. We need it in the lattice regularization. Since the potential is normally defined in terms of a non-local object, the Wilson loop, it is not obvious that $\mathrm{O}(a)$ improvement holds. But one may relate the potential exactly to correlators of local fields in the Heavy Quark Effective Theory [25] and thus concludes that $\mathrm{O}(a)$ improvement does hold for differences of potentials [25]. For a pedagogical description see [26]. In order 
to have an $\mathrm{O}(a)$ improved force one then just has to use a proper definition of the derivative. The most natural choice is $F_{\text {naive }}\left(r_{\text {naive }}\right)=\frac{1}{a}[V((r, 0,0))-V((r-a, 0,0))]$ with $r_{\text {naive }}=r-\frac{a}{2}$, when one uses the on-axis potential as we do here. However, it is much better to define the force such that it has no cutoff effects at the lowest order in perturbation theory as in eq. (2.9). This may be achieved by a different choice of the argument [27,

$$
F\left(r_{\mathrm{I}}\right)=\frac{1}{a}[V((r, 0,0))-V((r-a, 0,0))]=g_{0}^{2} \frac{C_{\mathrm{F}}}{4 \pi r_{\mathrm{I}}^{2}}+\mathrm{O}\left(g_{0}^{4}\right),
$$

with $C_{\mathrm{F}}=\left(N_{c}^{2}-1\right) / 2 N_{c}$. The improved radius $r_{\mathrm{I}}$ is computed from the tree-level potential

$$
V_{\text {tree }}(r)=\frac{1}{a} C_{\mathrm{F}} \int_{-\pi}^{\pi} \frac{\mathrm{d}^{3} k}{(2 \pi)^{3}} \frac{2 \sin ^{2}\left(\frac{k_{1}}{2} \frac{r}{a}\right)}{4 \sum_{j=1}^{3} \sin ^{2}\left(\frac{k_{j}}{2}\right)},
$$

by $r_{\mathrm{I}}^{-2}=4 \pi C_{\mathrm{F}}^{-1} \frac{1}{a}\left[V_{\text {tree }}((r, 0,0))-V_{\text {tree }}((r-a, 0,0))\right]$. See Ref. 25] for an efficient way of computing eq. (3.2). In the same reference, one can also see that non-perturbatively in the pure $\mathrm{SU}(3)$ gauge theory, the force defined in terms of $r_{\mathrm{I}}$ has much smaller lattice artifacts compared to $r_{\text {naive }}$. We will use eq. (3.1) in all numerical results that we show below. We have, however, looked at all quantities also for $r_{\text {naive }}$. There are no changes which are worth reporting about.

Loop corrections to the force are computed as described in [17]. We shall comment on details below. For now we write down immediately the expression renormalized in the $\overline{\mathrm{MS}}$ scheme and set the renormalization scale to the natural scale of the observable, $\mu=1 / r$.

$$
F=\frac{C_{\mathrm{F}} \alpha_{\overline{\mathrm{MS}}}(1 / r)}{r^{2}}\left\{1+f_{1}(\mathbf{z}, a / r) \alpha \overline{\mathrm{MS}}(1 / r)+\mathrm{O}\left(\alpha_{\overline{\mathrm{MS}}}^{2}\right)\right\}
$$

From here on we have $r=r_{\mathrm{I}}$ as the argument of the force and quantities derived from it without indicating the subscript "I" explicitly. The correction term is split into gluonic and fermionic contributions

$$
f_{1}=f_{1, g}(a / r)+\sum_{i=1}^{N_{f}} f_{1, f}\left(z_{i}, a / r\right) .
$$

For the determination of the lattice artifacts, we subtracted the continuum expressions $\left(\gamma_{E}=0.57721566 \ldots\right.$ is the Euler-Mascheroni constant)

$$
\begin{aligned}
f_{1, g}(0) & =\frac{N_{\mathrm{c}}}{\pi}\left[-\frac{35}{36}+\frac{11}{6} \gamma_{E}\right] \\
f_{1, f}(z, 0) & =\frac{1}{2 \pi}\left[\frac{1}{3} \log \left(z^{2}\right)+\frac{2}{3} \int_{1}^{\infty} \mathrm{d} x \frac{1}{x^{2}} \sqrt{x^{2}-1}\left(1+\frac{1}{2 x^{2}}\right)(1+2 z x) e^{-2 z x}\right],
\end{aligned}
$$




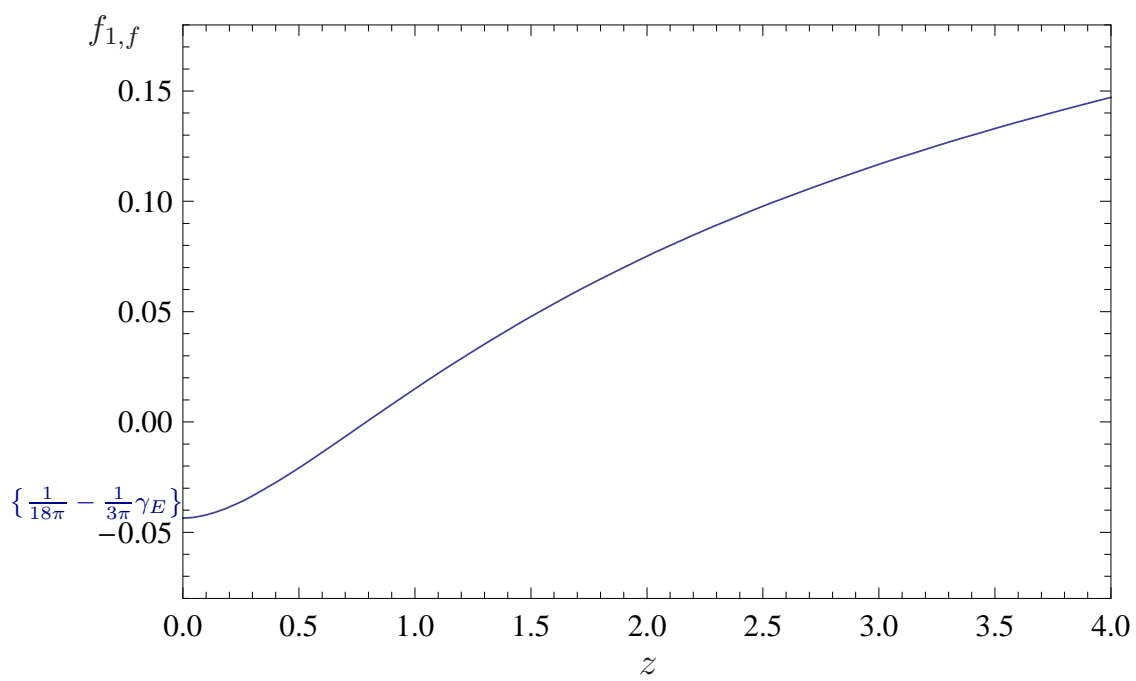

Figure 1: The one-loop fermionic force contribution $f_{1, f}(z, 0)$.

which are derived from the ones for the potential [28 31]. The integral in eq. (3.6) is evaluated numerically. We show the fermion contribution in Fig. 1.

The gluonic correction at finite $a / r$ is not our main concern. For reference we will just cite numbers from [32]. However, we performed a re-evaluation of $f_{1, f}(z, a / r)$ following Ref. [17. For each value of $r / a$ the potential is given by a seven-dimensional integral over the Brillouin zone. At fixed $a m_{0}$ and $r / a$ we evaluated these momentum integrals by discretizing the Brillouin zone with a regular momentum lattice with spacing $\Delta k=2 \pi / l$, applying the trapezoidal rule. This procedure was carried out for $r / a \in$ $[1.358,15.467]$ and for more than thirty values of $a m_{0} \in[-0.05,1.5]$. The force was then extrapolated in the momentum spacings, i.e. we took the limit $l \rightarrow \infty$. It is advantageous to extrapolate the force and not the potential since the large unphysical self-energy contribution is then avoided. The numerical results for the force at fixed $r / a$ were then interpolated in $a m_{0}$, and the interpolations were subsequently used at the desired values of $z$. Interpolation and extrapolation errors form the uncertainties visible in our figures below.

Following our definition of the relative lattice artifacts, we have

$$
\begin{aligned}
\left.\frac{F-F_{\text {cont }}}{F_{\text {cont }}}\right|_{\bar{g}_{\overline{\mathrm{MS}}},\left(m_{i}\right)_{\mathrm{R}}} & =\delta_{F}^{(1)}(\mathbf{z}, a / r) \bar{g}_{\overline{\mathrm{MS}}}^{2}(1 / r), \\
\delta_{F}^{(1)}(\mathbf{z}, a / r) & =\delta_{F}^{(1, g)}(a / r)+\sum_{i=1}^{N_{\mathrm{f}}} \delta_{F}^{(1, f)}\left(z_{i}, a / r\right),
\end{aligned}
$$

and, for example,

$$
4 \pi \delta_{F}^{(1, f)}(z, a / r)=f_{1, f}(z, a / r)-f_{1, f}(z, 0) .
$$




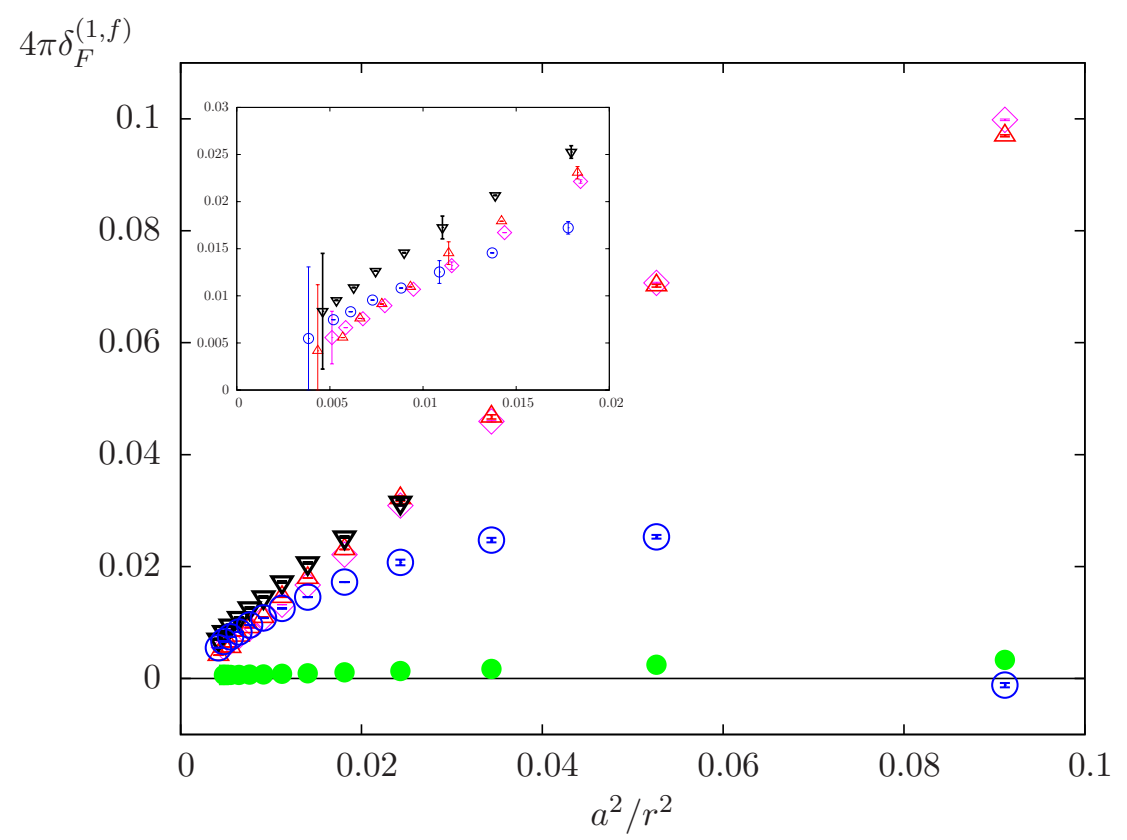

Figure 2: The fermionic cutoff effects $4 \pi \delta_{F}^{(1, f)}(z, a / r)$. For the improved theory $\left(c_{\mathrm{sw}}=1\right.$, $\left.b_{\mathrm{g}}=0.01200(2)\right)$ and our standard choice $\left(m_{i}\right)_{\mathrm{R}}=\widetilde{m}_{\mathrm{q}, i}$ we show $z=0(\diamond), z=1(\triangle)$ and $z=3(\nabla)$ which is restricted to the range $a\left(m_{i}\right)_{\mathrm{R}}<1 / 2$. For the pole mass definition we display just $z=3(\bigcirc)$. Mass-less quarks with $c_{\mathrm{sw}}=b_{\mathrm{g}}=0$ are given by $\bullet$ We show some typical errors resulting from the $l$-extrapolation and the interpolation in $a m_{0}$.

The factor $4 \pi$ means that the relative size of the cutoff effect is given by multiplying with $\alpha=\bar{g}^{2} /(4 \pi)$.

A first impression on the relevant overall size of lattice artifacts is gained from the gluonic piece $\delta_{F}^{(1, g)}(a / r)$. The results of Ref. 32] are not precise enough to see the asymptotic decay in $(a / r)^{2}$. Instead, we just extract

$$
\begin{aligned}
& 4 \pi \delta_{F}^{(1, g)}(a / r)=-0.232(6) \text { for } r / a=2.277, \\
& 4 \pi \delta_{F}^{(1, g)}(a / r)=-0.190(19) \text { for } r / a=3.312, \\
& 4 \pi \delta_{F}^{(1, g)}(a / r)=-0.151(42) \text { for } r / a=4.319,
\end{aligned}
$$

from the numbers listed in Ref. [32]. For the naive definition $\left(r_{\text {naive }}\right)$ of the lattice force, the cutoff effects are about a factor of five larger and have the opposite sign compared to (3.10). Perturbation theory is probably a good guideline up to $\alpha=1 / 3$ at which point we are then looking at around $5 \%$ effects - perturbation theory indicates that larger values of $r / a$ are needed for precision physics.

In Fig. 2 we display some of our results for the fermionic contribution to the cutoff effects $4 \pi \delta_{F}^{(1, g)}$. Those following exactly our description of $\mathrm{O}(a)$-improvement $\left(c_{\mathrm{Sw}}=1\right)$ and renormalization $\left(\left(m_{i}\right)_{\mathrm{R}}=\widetilde{m}_{\mathrm{q}, i}\right)$ are shown by open symbols. The scale on the y-axis 
is about a factor two smaller than the pure gauge effects eq. 3.10). Generically the fermionic cutoff effects are smaller than the gluonic ones and they depend very little on the mass.

For illustration, we include in Fig. 2 also a different renormalization of the quark mass. In perturbation theory, a possible one is the "pole mass", $\left(m_{i}\right)_{\mathrm{R}}=\frac{1}{a} \log (1+$ $\left.a m_{0, i}\right)+\mathrm{O}\left(g_{0}^{2}\right) 5$ This mass can be taken larger in lattice units, which we do here only to expose the resulting problematic cutoff effects. While these happen to be smaller than the cutoff effects for $\left(m_{i}\right)_{\mathrm{R}}=\widetilde{m}_{\mathrm{q}, i}$, they have a rather non-linear behaviour as a function of $a^{2} / r^{2}$. We further remark that the asymptotic $a^{2} / r^{2}$ scaling sets in later when $z$ is increased for this case.

A second remark concerns mass-less quarks discretized with $c_{\mathrm{sw}}=0$. For massless quarks automatic $\mathrm{O}(a)$ improvement holds in perturbation theory for any value of $c_{\mathrm{Sw}}$ [33] (see [34] for a simple proof). The expected form of the cutoff effects is seen in the figure and their magnitude happens to be smaller than for $c_{\mathrm{sw}}=1$.

Next, let us come back to the overall magnitude. Together with the factor $\alpha$ which accompanies $4 \pi \delta_{F}^{(1, f)}$, the magnitude is small. One has to remember that, since we consider a relative cutoff effect, it is normalized to the leading order, $N_{\mathrm{f}}$-independent, term. However, the whole fermionic contribution is relatively small. So in comparison to the physical effect of the fermions, their cutoff effects are noticeable. Of course the continuum fermionic vacuum polarization contribution depends on how one renormalizes the theory. In $\overline{\mathrm{MS}}$-renormalization, its smallness is just seen in Fig. 1, and in the nonperturbatively defined SF-scheme (see the following section), one has an even smaller one-loop coefficient $f_{1, f}^{\mathrm{SF}}$ in the relation $F=\frac{C_{\mathrm{F}} \alpha_{\mathrm{SF}}(1 / r)}{r^{2}}\left\{1+\left[f_{1, g}^{\mathrm{SF}}+N_{\mathrm{f}} f_{1, f}^{\mathrm{SF}}\right] \alpha_{\mathrm{SF}}(1 / r)+\ldots\right\}$ for massless quarks. Interestingly enough, the situation is reversed when we consider the mass-dependence. The physical effect, the variation in Fig. 1, is of the order of 0.2, while the cutoff effect is the difference of the data marked as triangles and the massless ones marked as diamonds. These cutoff effects are tiny compared to the physical ones.

So far we have discussed the cutoff effects in a massless renormalization scheme. We may define a massive scheme by a coupling $\bar{g}_{m}^{2}=\frac{4 \pi r_{0}^{2}}{C_{\mathrm{F}}} F\left(r_{0}\right)$ and then look at fixed $r_{0} \mathbf{m}_{\mathrm{R}}$ and $r / r_{0}$ how the continuum limit is approached. Making use of eq. (2.21) the contribution of a massive quark to the relative cutoff effects in $F(r)$ is then $\left[\delta_{F}^{(1, f)}\left(r m_{\mathrm{R}}, a / r\right)-\delta_{F}^{(1, f)}\left(r_{0} m_{\mathrm{R}}, a / r_{0}\right)\right] \bar{g}_{\mathrm{m}}^{2}\left(1 / r_{0}, \mathbf{m}_{\mathrm{R}}\right)+\mathrm{O}\left(g^{4}\right)$. Since $\delta_{F}^{(1, f)}(z, a / r)$ depends hardly on $z$, such cutoff effects are small as well as long as $a / r_{0}$ is small.

\footnotetext{
${ }^{5}$ We note that there is a non-perturbative definition which coincides with the pole mass at the lowest order of perturbation theory, which is relevant here. The non-perturbative mass is $m_{p}=$ $-\frac{\mathrm{d}}{\mathrm{d} x_{0}} \log \left(f_{\mathrm{P}}\left(x_{0}\right)\right)$, with the Schrödinger functional correlator $f_{\mathrm{P}}\left(x_{0}\right)$ as defined in Ref. [16] for $\theta=0$ and without background field.
} 


\section{The Schrödinger functional coupling}

The Schrödinger functional is the field theory in a finite space-time, taken here as $L^{4}$, with Dirichlet boundary conditions in time and periodic boundary condition (up to a phase $\theta$ for the quark fields) in space [15, 24, 35]. The action can be written exactly as in Sect. 2 if the fields outside the interval $0 \leq x_{0} \leq L$ are set to zero, the gauge links on the boundaries $x_{0}=0$ and $x_{0}=L$ are set to fixed values and the fermion fields on the boundary are set to zero. Symanzik's effective action now contains terms localized at the boundaries with new couplings. These result in cutoff effects linear in the lattice spacing which are canceled by four more improvement coefficients. One of them modifies the weight of the plaquettes touching the boundary to $w(p)=c_{\mathrm{t}}=$ $1-\left[0.08900+0.019141 N_{\mathrm{f}} \pm 0.00005\right] g_{0}^{2}+\ldots$, the other ones play no rôle in our one-loop computation due to our choice of the boundary conditions (abelian background field point "A" [24]) and due to the order in $g_{0}^{2}$. For more details we refer to Refs. [15, 16, 24,

The main virtue of the Schrödinger functional is that it can be simulated for massless quarks and a running coupling can be defined and computed precisely in a Monte Carlo simulation (see [36] for a review). We take the definition of the coupling from Refs. [15,24]. It depends on $L$ which plays the rôle of an inverse renormalization scale, on the angle $\theta$ introduced above, and on a dimensionless parameter $\nu$ which is usually set to zero. The dependence on $\nu$ is explicit,

$$
\frac{1}{\bar{g}_{\nu}^{2}(L, \nu, \mathbf{z})}=\frac{1}{\bar{g}_{\mathrm{SF}}^{2}(L, \mathbf{z})}-\nu \bar{v}(L, \mathbf{z}) .
$$

We consider $\bar{g}_{\mathrm{SF}}$ and the quantity $\bar{v}$ which vanishes at tree-level. The mass-dependence is parameterized by the dimensionless $z_{i}=\left(m_{i}\right)_{\mathrm{R}} L$.

The central object needed in the non-perturbative computation of the running of the coupling is the so-called step scaling function,

$$
\left.\Sigma(u, \mathbf{z}, a / L) \equiv \bar{g}_{\mathrm{SF}}^{2}(2 L, 2 z)\right|_{\bar{g}_{\mathrm{SF}}^{2}(L, \mathbf{z})=u,\left(m_{i}\right)_{\mathrm{R}} L=z_{i}}=u+\Sigma_{1}(\mathbf{z}, a / L) u^{2}+\ldots,
$$

but to see how cutoff effects behave we can also look at other quantities, in particular

$$
\left.\Omega(u, \mathbf{z}, a / L) \equiv \bar{v}(L, \mathbf{z})\right|_{\bar{g}_{\mathrm{SF}}^{2}(L, \mathbf{z})=u,\left(m_{i}\right)_{\mathrm{R}} L=z_{i}}=\bar{v}_{1}(\mathbf{z}, a / L)+\mathrm{O}(u),
$$

and

$$
\left.\rho(u, \mathbf{z}, a / L) \equiv \frac{\bar{g}_{\mathrm{SF}}^{2}(L, \mathbf{z})-\bar{g}_{\mathrm{SF}}^{2}(L, \mathbf{0})}{\bar{g}_{\mathrm{SF}}^{2}(L, \mathbf{0})}\right|_{\bar{g}_{\mathrm{SF}}^{2}(L, 0)=u,\left(m_{i}\right)_{\mathrm{R}} L=z_{i}}=\rho_{1}(\mathbf{z}, a / L) u+\mathrm{O}\left(u^{2}\right) .
$$

For the step scaling function $\Sigma$, we consider the relative lattice artifacts, defined as in eq. (2.18), with the expansion

$$
\delta_{\Sigma}(u, \mathbf{z}, a / L)=\frac{\Sigma(u, \mathbf{z}, a / L)-\Sigma(u, \mathbf{z}, 0)}{\Sigma(u, \mathbf{z}, 0)}=\delta_{\Sigma}^{(1)}(\mathbf{z}, a / L) u+\ldots
$$




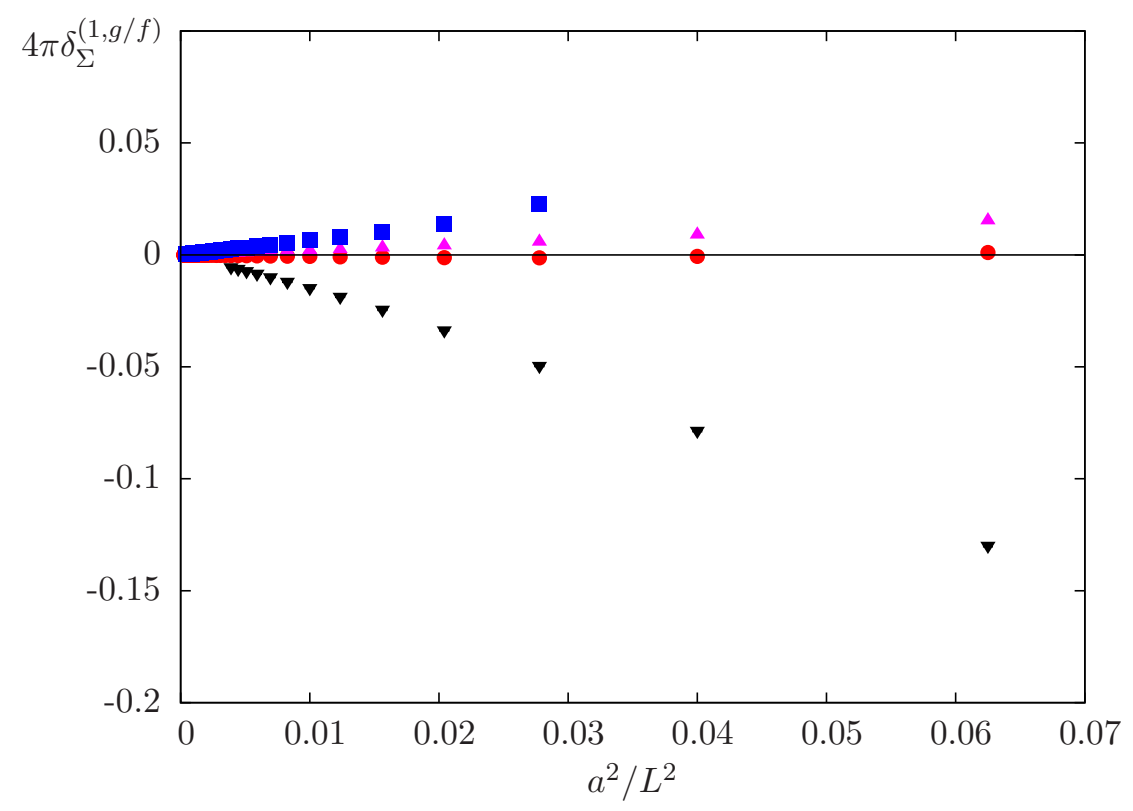

Figure 3: The cutoff effects $4 \pi \delta_{\Sigma}^{(1, g)}(a / L)(\boldsymbol{\nabla})$ and $4 \pi \delta_{\Sigma}^{(1, f)}(z, a / L)$ for $z=0(\bullet)$, $z=1(\boldsymbol{\Delta})$ and $z=3(\boldsymbol{\square})$ extracted for $\theta=\pi / 5$.

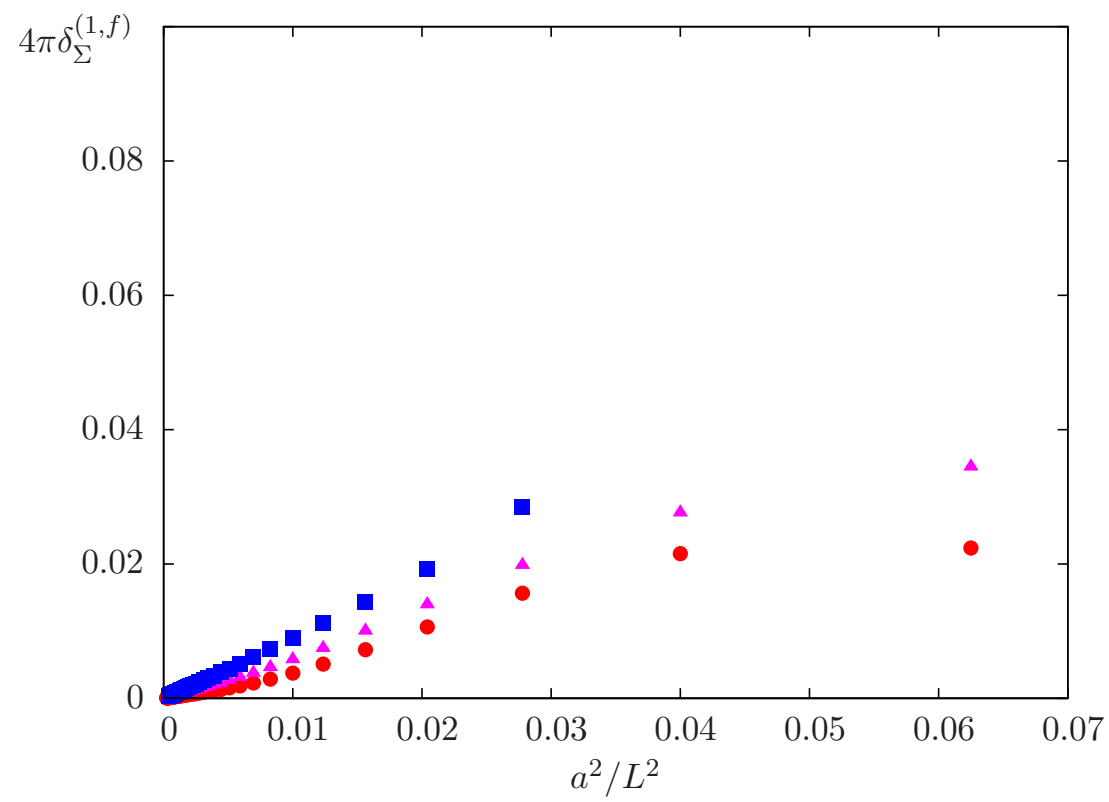

Figure 4: The cutoff effects $4 \pi \delta_{\Sigma}^{(1, f)}(z, a / L)$ for $z=0(\bullet), z=1(\boldsymbol{\Delta})$ and $z=3$ extracted for $\theta=0$. 


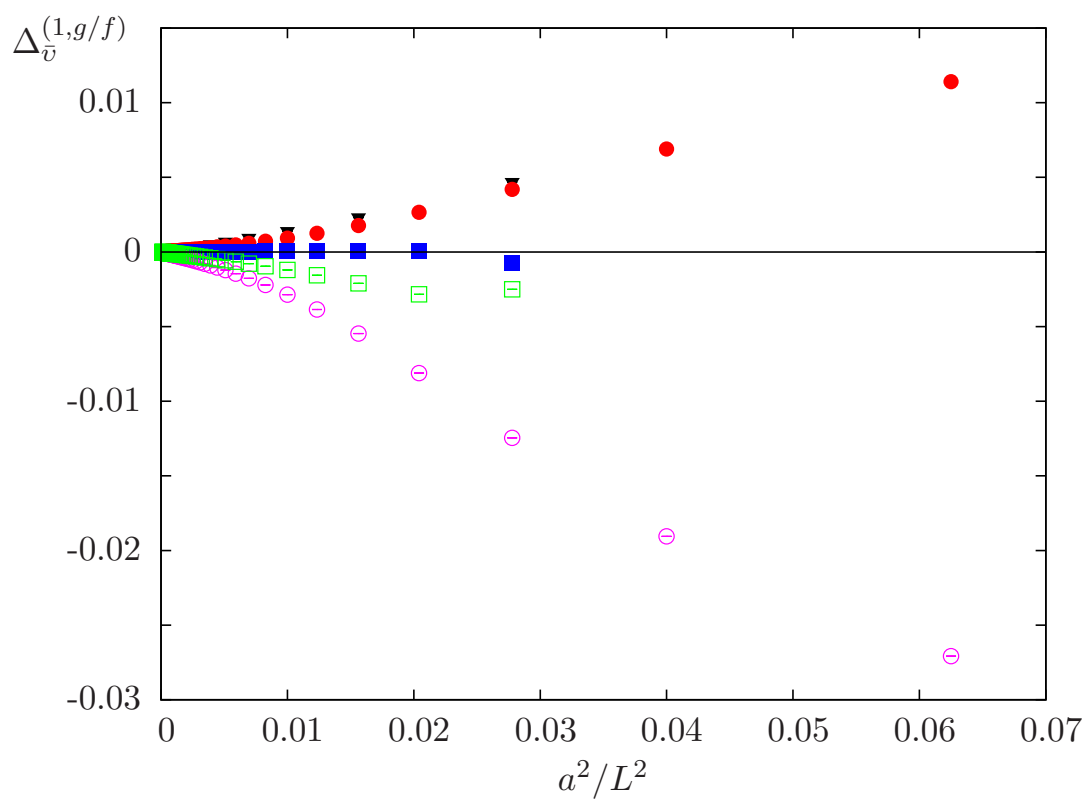

Figure 5: The cutoff effects $\Delta_{\bar{v}}^{(1, g)}(a / L)(\boldsymbol{\nabla})$ and $\Delta_{\bar{v}}^{(1, f)}(z, a / L)$ for $z=0(\bullet(\bigcirc))$ and $z=3(\square(\square))$ extracted for $\theta=\pi / 5(\theta=0)$.

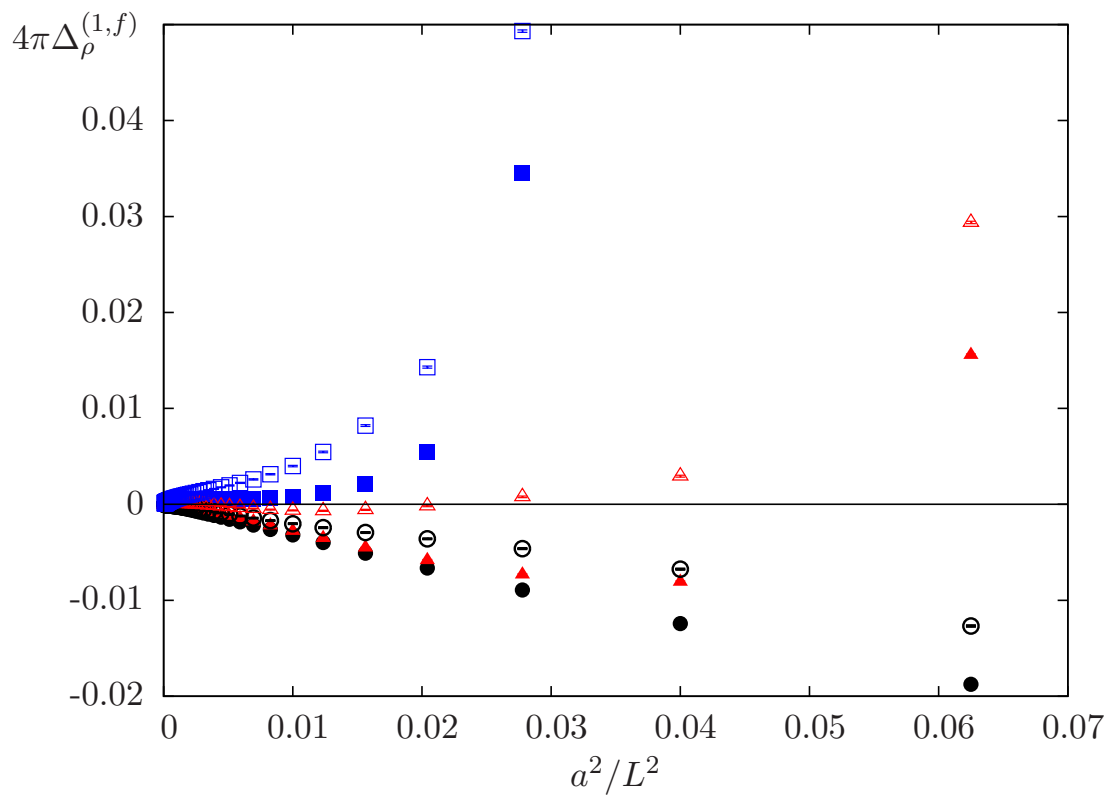

Figure 6: The cutoff effects $4 \pi \Delta_{\rho}^{(1, f)}(z, a / L)$ for $z=1(\bullet(\bigcirc)), z=2(\boldsymbol{\Delta}(\triangle))$ and $z=3(\square(\square))$ extracted for $\theta=\pi / 5(\theta=0)$. 
However, since both $\rho$ and $\bar{v}$ vanish at tree level it is more convenient to consider the absolute artifacts,

$$
\begin{aligned}
& \Delta_{\bar{v}}(u, \mathbf{z}, a / L) \equiv \Omega(u, \mathbf{z}, a / L)-\Omega(u, \mathbf{z}, 0)=\Delta_{\bar{v}}^{(1)}(\mathbf{z}, a / L)+\mathrm{O}(u) \\
& \Delta_{\rho}(u, \mathbf{z}, a / L) \equiv \rho(u, \mathbf{z}, a / L)-\rho(u, \mathbf{z}, 0)=\Delta_{\rho}^{(1)}(\mathbf{z}, a / L) u+\mathrm{O}\left(u^{2}\right)
\end{aligned}
$$

Their decomposition into gluonic and fermionic pieces reads

$$
\begin{aligned}
\delta_{\Sigma}^{(1)}(\mathbf{z}, a / L) & =\delta_{\Sigma}^{(1, g)}(\mathbf{z}, a / L)+\sum_{i=1}^{N_{\mathrm{f}}} \delta_{\Sigma}^{(1, f)}\left(z_{i}, a / L\right), \\
\Delta_{\bar{v}}^{(1)}(\mathbf{z}, a / L) & =\Delta_{\bar{v}}^{(1, g)}(a / L)+\sum_{i=1}^{N_{\mathrm{f}}} \Delta_{\bar{v}}^{(1, f)}\left(z_{i}, a / L\right), \\
\Delta_{\rho}^{(1)}(\mathbf{z}, a / L) & =\sum_{i=1}^{N_{\mathrm{f}}} \Delta_{\rho}^{(1, f)}\left(z_{i}, a / L\right) .
\end{aligned}
$$

We note that $\Delta_{\rho}^{(1)}$ is a cutoff effect in a massless renormalization scheme (see Sect. 2.1), while $\delta_{\Sigma}^{(1)}, \Delta_{\bar{v}}^{(1)}$ refer to the massive Schrödinger functional renormalization scheme (see Sect. 2.2.2).

The artifact $\delta_{\Sigma}^{(1)}(\mathbf{z}, a / L)$ is illustrated in Fig. 3 for $\theta=\pi / 5$ and for $\theta=0$ in Fig. 46 One observes that the cutoff effects for an individual $\mathrm{O}(a)$ improved fermion are significantly smaller than the gluonic piece. For $\theta=\pi / 5$, massless $\mathrm{O}(a)$ improved fermions hardly show any cutoff effect - a kinematical accident. Generically cutoff effects grow with $z$, but not very much. Of course, one should not forget that for several fermion flavors they add up accordingly.

Compared to the step scaling function, the cutoff effects in $\bar{v}$, Fig. 5 , are bigger, in particular when one takes into account that the relevant overall magnitude is $\bar{v}_{1} \approx 0.1$ (that number varies somewhat with the number of flavors and the parameter $\theta$ ). Massive quarks show smaller effects than massless ones.

Finally, Fig. 6 shows the cutoff effects in $\rho$. These are to be compared to the continuum values of $4 \pi \rho(z, 0)$ which range from 0.095 (0.086) for $z=1$ to $0.188(0.170)$ for $z=3$ at $\theta=\pi / 5(\theta=0)$.

\section{Conclusions}

In one-loop lattice perturbation theory, we investigated cutoff effects from including $\mathrm{O}(a)$-improved Wilson quarks, and observed that in certain cases they are comparable to the cutoff effects in the pure gauge theory, see Fig. 5. However, our main motivation for studying these was to check whether they become large for massive quarks, in particular

\footnotetext{
${ }^{6}$ The choice $\theta=\pi / 5$ is the standard, since it was seen to be advantageous for Monte Carlo computations [15, while $\theta=0$ is a natural alternative.
} 


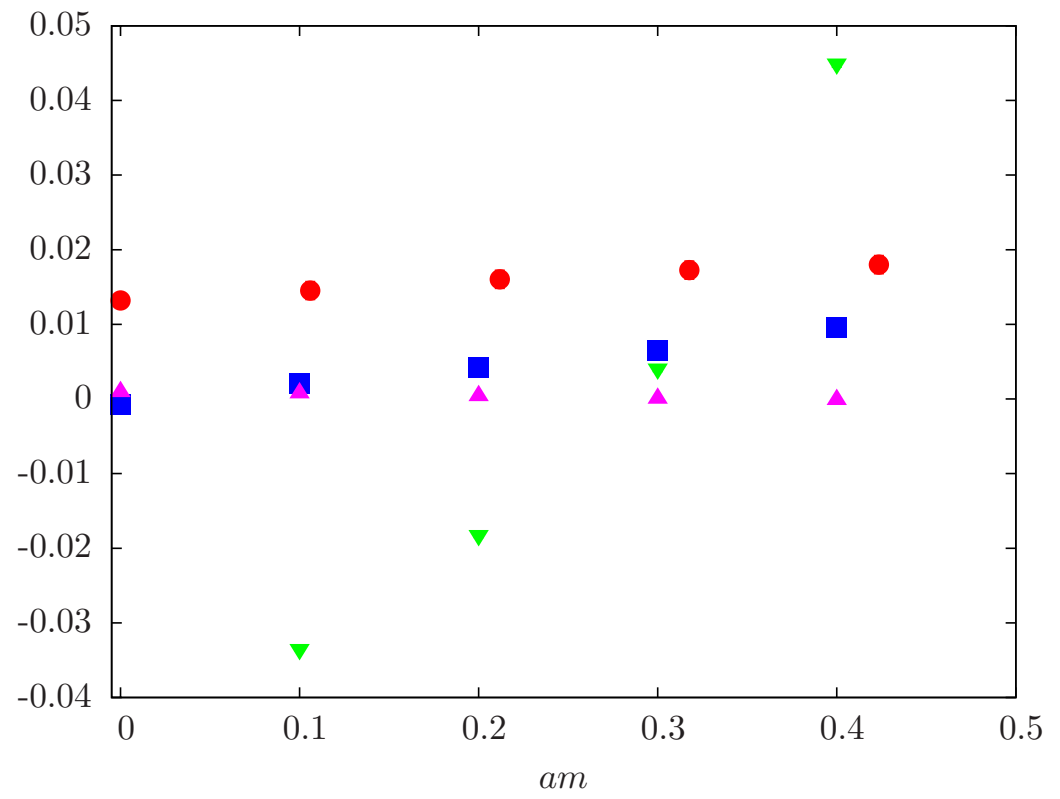

Figure 7: Mass dependence of a few cutoff effects. We fix $a / r=1 / 9.445, a / L=1 / 10$ and show $4 \pi \delta_{F}^{(1, f)}(\bullet)$ as well as $4 \pi \delta_{\Sigma}^{(1, f)}(\boldsymbol{\square}) \Delta_{\bar{v}}^{(1, f)}(\boldsymbol{\Delta}) \Delta_{\rho}^{(1, f)} / \rho^{(1, f)}(z, 0)$ ( $\left.\boldsymbol{\nabla}\right)$ for $\theta=\pi / 5$. In contrast to our other graphs, the limit $a m \rightarrow 0$ does not correspond to the continuum limit since here we keep $a / L$ fixed and not $z$.

for values of $a m=1 / 4-1 / 2$ which may be encountered in simulations with a charm quark.

Contrary to expectation, we find that the lattice artifacts do not grow much and sometimes even become smaller for larger masses. This statement is further illustrated in Fig. 7 where we choose a good enough resolution to have small artifacts for massless quarks and then display them as a function of the mass in lattice units. They do remain small, which provides a good indication that dynamical charm quarks do not distort lattice QCD simulations at typical presently used lattice spacings.

\section{Acknowledgements.}

We thank Haris Panagopoulos and Ulli Wolff for useful discussions, Hubert Simma for providing valuable comments on the manuscript and Mathias Steinhauser for pointing out Ref. [31. This work is supported by the Deutsche Forschungsgemeinschaft in the SFB/TR 09. Computations were carried out on the compute farm at DESY, Zeuthen. 


\section{References}

[1] MILC collaboration Collaboration, A. Bazavov et. al., Scaling studies of QCD with the dynamical HISQ action, Phys.Rev. D82 (2010) 074501, [arXiv:1004.0342].

[2] R. Baron, P. Boucaud, J. Carbonell, A. Deuzeman, V. Drach, et. al., Light hadrons from lattice QCD with light $(u, d)$, strange and charm dynamical quarks, JHEP 1006 (2010) 111, arXiv:1004.5284.

[3] ALPHA Collaboration, F. Tekin, R. Sommer, and U. Wolff, The Running coupling of QCD with four flavors, Nucl.Phys. B840 (2010) 114-128, [1006.0672].

[4] G. Herdoiza, Towards Four-Flavour Dynamical Simulations, PoS

LATTICE2010 (2010) 010, arXiv:1103.1523.

[5] M. Lüscher, P. Weisz, and U. Wolff, A numerical method to compute the running coupling in asymptotically free theories, Nucl. Phys. B359 (1991) 221-243.

[6] M. Lüscher, R. Sommer, P. Weisz, and U. Wolff, A precise determination of the running coupling in the SU(3) Yang-Mills theory, Nucl. Phys. B413 (1994) 481-502, hep-lat/9309005.

[7] ALPHA Collaboration, S. Capitani, M. Lüscher, R. Sommer, and H. Wittig, Non-perturbative quark mass renormalization in quenched lattice QCD, Nucl. Phys. B544 (1999) 669, hep-lat/9810063.

[8] ALPHA Collaboration, M. Della Morte et. al., Computation of the strong coupling in QCD with two dynamical flavours, Nucl. Phys. B713 (2005) 378-406, hep-lat/0411025.

[9] PACS-CS Collaboration, S. Aoki et. al., Precise determination of the strong coupling constant in $N(f)=2+1$ lattice $Q C D$ with the Schrodinger functional scheme, JHEP 0910 (2009) 053, 0906.3906.

[10] ALPHA Collaboration, J. Rolf and S. Sint, A precise determination of the charm quark's mass in quenched QCD, JHEP 12 (2002) 007, hep-ph/0209255.

[11] ALPHA Collaboration, A. Jüttner and J. Rolf, A precise determination of the decay constant of the $\mathrm{d} / \mathrm{s}$ - meson in quenched QCD, Phys. Lett. B560 (2003) 59-63, hep-lat/0302016.

[12] J. Heitger and A. Jüttner, Lattice cutoff effects for $F_{D_{s}}$ with improved Wilson fermions - a final lesson from the quenched case, JHEP 05 (2009) 101, [arXiv:0812.2200]. 
[13] ALPHA Collaboration, M. Kurth and R. Sommer, Heavy quark effective theory at one-loop order: An explicit example, Nucl. Phys. B623 (2002) 271-286, hep-lat/0108018.

[14] ALPHA Collaboration, J. Heitger and J. Wennekers, Effective heavy-light meson energies in small-volume quenched qcd, JHEP 02 (2004) 064, hep-lat/0312016.

[15] S. Sint and R. Sommer, The running coupling from the QCD Schrödinger functional: A one loop analysis, Nucl. Phys. B465 (1996) 71-98, hep-lat/9508012.

[16] M. Lüscher, S. Sint, R. Sommer, and P. Weisz, Chiral symmetry and $O(a)$ improvement in lattice QCD, Nucl. Phys. B478 (1996) 365-400, hep-lat/9605038.

[17] A. Athenodorou and H. Panagopoulos, Large Wilson loops with overlap and clover fermions: Two-loop evaluation of the b-quark mass shift and the quark-antiquark potential, Nucl.Phys. B799 (2008) 1-18, hep-lat/0509039.

[18] T. Bhattacharya, R. Gupta, W. Lee, S. R. Sharpe, and J. M. Wu, Improved bilinears in lattice QCD with non-degenerate quarks, Phys.Rev. D73 (2006) 034504, hep-lat/0511014.

[19] B. Sheikholeslami and R. Wohlert, Improved continuum limit lattice action for QCD with Wilson fermions, Nucl. Phys. B259 (1985) 572.

[20] R. F. Dashen and D. J. Gross, The Relationship Between Lattice and Continuum Definitions of the Gauge Theory Coupling, Phys.Rev. D23 (1981) 2340.

[21] A. Hasenfratz and P. Hasenfratz, The Scales of Euclidean and Hamiltonian Lattice QCD, Nucl.Phys. B193 (1981) 210.

[22] M. Lüscher and P. Weisz, Two loop relation between the bare lattice coupling and the $M S$ coupling in pure $S U(N)$ gauge theories, Phys. Lett. B349 (1995) 165-169, hep-lat/9502001.

[23] A. Bode and H. Panagopoulos, The three-loop beta-fuction of QCD with the clover action, Nucl. Phys. B625 (2002) 198-210, hep-lat/0110211.

[24] M. Lüscher, R. Narayanan, P. Weisz, and U. Wolff, The Schrödinger functional: A renormalizable probe for non-Abelian gauge theories, Nucl. Phys. B384 (1992) 168-228, hep-lat/9207009.

[25] S. Necco and R. Sommer, The $N(f)=0$ heavy quark potential from short to intermediate distances, Nucl. Phys. B622 (2002) 328-346, http://arXiv.org/abs/hep-lat/0108008. 
[26] R. Sommer, Introduction to Non-perturbative Heavy Quark Effective Theory, arXiv:1008.0710, Lectures at the Summer School on "Modern perspectives in lattice QCD", Les Houches, August 3-28, 2009.

[27] R. Sommer, A new way to set the energy scale in lattice gauge theories and its applications to the static force and $\alpha_{s}$ in SU(2) Yang-Mills theory, Nucl. Phys. B411 (1994) 839, hep-lat/9310022.

[28] W. Fischler, Quark - anti-Quark Potential in QCD, Nucl.Phys. B129 (1977) $157-174$.

[29] A. Billoire, How Heavy Must Be Quarks in Order to Build Coulombic q anti-q Bound States, Phys.Lett. B92 (1980) 343.

[30] M. Melles, The Static QCD potential in coordinate space with quark masses through two loops, Phys.Rev. D62 (2000) 074019, hep-ph/0001295.

[31] A. Hoang, Bottom quark mass from Upsilon mesons: Charm mass effects, hep-ph/0008102.

[32] G. S. Bali and P. Boyle, Perturbative Wilson loops with massive sea quarks on the lattice, hep-lat/0210033.

[33] R. Frezzotti and G. C. Rossi, Chirally improving Wilson fermions. i: $O(a)$ improvement, JHEP 08 (2004) 007, hep-lat/0306014.

[34] S. Sint, Lattice QCD with a chiral twist, In Perspectives in Lattice QCD, World Scientific 2008 (2007) hep-lat/0702008.

[35] S. Sint, On the Schrödinger functional in QCD, Nucl. Phys. B421 (1994) 135-158, hep-lat/9312079.

[36] R. Sommer, Non-perturbative QCD: Renormalization, O(a)-improvement and matching to heavy quark effective theory, In Perspectives in Lattice QCD, World Scientific 2008 (2006) hep-lat/0611020. 